\title{
On the Nature of Irregular Variables of Type Lb
}

\author{
FRANZ KERSCHBAUM ${ }^{1}$, PETER HABISON ${ }^{1,2}$, \\ and JOSEF HRON ${ }^{1}$
}

\author{
${ }^{1}$ Institut für Astronomie der Universität Wien, Vienna, Austria \\ ${ }^{2}$ Kuffner-Sternwarte, Vienna, Austria
}

The study deals with one group of stars at the low-mass-loss part of the asymptotic giant branch, namely the Irregular variables of type $\mathrm{Lb}$. Their distinctly different pulsational behaviour, when compared with the well studied Miras, combined with the presumed importance of pulsation for the stellar mass loss, make them important scientific targets. O-rich $\mathrm{Lb}$ variables were selected from the GCVS and the IRAS Catalogue. Additional near infrared data was used, mainly from our own observations. Comparison samples of Miras, SRa-, and SRb-type variables were selected in the same way.

For the four groups of O-rich variables, pulsational properties like the amplitude or the period (not for Lbs) are correlated with atmospheric properties like the effective temperature and can be used to separate at least Lbs, SRas and SRbs from the Miras. By means of NIR as well as IRAS two-color diagrams, O-rich Lbs are indistinguishable from SRas or SRbs. Hence, it seems likely that they have similar effective temperatures, atmospheric structure and also mass-loss rates. The Lbs fit nicely in the areas of "blue" and "red" SRVs. But additionally one finds a few objects displaying NIR properties similar to $M$ giants. These stars may be not on the AGB at all. All three groups are well separated from the Miras. Also the blackbody-fit results turn out to be comparable for the Lb, SRa, and SRb stars and different for the Miras (for SRVs see $A \& A 308,489,1996$ ). Whereas the first three have relatively high photospheric temperatures $\mathrm{T}^{*}$, medium dust temperatures $\mathrm{T}^{\mathrm{d}}$ and a wide spread in the relative sizes of the dust blackbody, the later tend towards lower $\mathrm{T}^{*}$ and somewhat higher $\mathrm{T}^{\mathrm{d}}$. This can be interpreted in the sense that Lbs, SRas and SRbs contain some stars with $60 \mu \mathrm{m}$ excess originating from colder dust and that Miras suffer higher mass loss with perhaps hotter dust temperatures. IRAS $60 \mu \mathrm{m}$ mass-loss rates are generally much higher for Miras and well correlated with some results of the $\mathrm{BB}$ fits, namely $\mathrm{T}^{*}$ and $\mathrm{L}^{\mathrm{d}} / \mathrm{L}^{*}$. The scale heights of Lbs and SRVs are quite similar, confirming our assumption of similar luminosities for these two groups.

This work is supported by the Fonds zur Förderung der wissenschaftlichen Forschung under project number S7308-AST. 